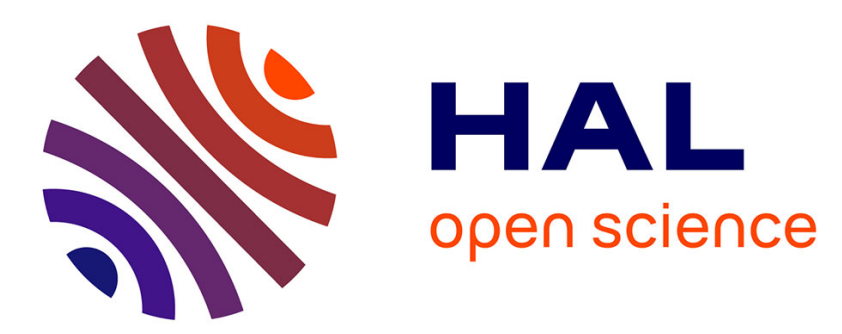

\title{
Extraction of mechanical work from stimuli-responsive molecular systems and materials
}

\author{
Alexis Perrot, Emilie Moulin, Nicolas Giuseppone
}

\section{To cite this version:}

Alexis Perrot, Emilie Moulin, Nicolas Giuseppone. Extraction of mechanical work from stimuliresponsive molecular systems and materials. Trends in Chemistry, 2021, 3 (11), pp.926-942. 10.1016/j.trechm.2021.08.007 . hal-03418854

\section{HAL Id: hal-03418854 \\ https://hal.science/hal-03418854}

Submitted on 8 Nov 2021

HAL is a multi-disciplinary open access archive for the deposit and dissemination of scientific research documents, whether they are published or not. The documents may come from teaching and research institutions in France or abroad, or from public or private research centers.
L'archive ouverte pluridisciplinaire HAL, est destinée au dépôt et à la diffusion de documents scientifiques de niveau recherche, publiés ou non, émanant des établissements d'enseignement et de recherche français ou étrangers, des laboratoires publics ou privés. 


\title{
Extraction of mechanical work from stimuli-responsive
}

\section{molecular systems and materials}

\author{
Alexis Perrot, Emilie Moulin, and Nicolas Giuseppone* \\ SAMS Research Group, Institut Charles Sadron, CNRS, University of Strasbourg, \\ 67000 Strasbourg, France
}

*Correspondence: giuseppone@unistra.fr

\section{Keywords}

Molecular switches, Molecular motors, Stimuli-responsive materials, Soft actuators

\begin{abstract}
Molecular switches and advanced molecular motors, which are the elementary building blocks for the construction of molecular machines, have been recently integrated into soft materials in order to generate macroscopic actuation under various types of external stimulations. However, to produce a continuous work from these materials, and therefore to potentially achieve more advanced tasks, important structural and dynamic aspects should be considered at all length scales. Here we discuss the implementation of thermodynamic, photodynamic, and dissipative molecular switches and motors in such stimuli-responsive materials. We also highlight the different ratcheting strategies that can be implemented in these actuators to confer them with the capacity of achieving unidirectional cyclic motion, and in order to continuously and autonomously increase their work output.
\end{abstract}


Towards a new generation of molecular systems and materials that can perform mechanical tasks

Machines convert an input of energy in mechanical forces and movement in order to perform a task. In our everyday life at macroscopic scale, we are acquainted with machines consuming electric or thermal energy for transport or automation. At nanoscale, living organisms also make use of biomolecular machines to function :[1] for instance, kinesin walkers or salmonella $\mathrm{H}^{+}$-driven motor use a chemical fuel (i.e. ATP) for the transportation of cargos across the cells or for bacterial locomotion.[2,3] In both macro- and nanoscale machines, the production of a unidirectional and repetitive mechanical actuation (such as a continuous rotation) is mandatory to constantly increase the work they can produce on their environment and to achieve complex tasks autonomously. However, if ratchetting devices are common at macroscale, reaching unidirectional motion in a Brownian environment is a real challenge which requires a deep thermodynamic control of the molecular actuation.

From a chemist point of view, the blossoming development of stimuli-responsive molecules and materials therefrom is of particular interest to think about the design of new kinds of mechanically active synthetic systems, capable of controlled actuation at different length scales by consuming various sources of energy.[4] Nevertheless, the amplification of nanoactuations up to the macroscopic scale is a difficult objective to reach because it requires to finely engineer the coupling of dynamic molecular elements in space and time. In addition, upon energy absorption, the mechanical work done by purely switching molecular actuators in devices and materials is usually undone when resetting them to their original state due to microscopic reversibility (see Glossary). Therefore, breaking spatial and time-reversal symmetries in such systems constitute an important challenge for the design of truly active materials.[5] In this direction, dissipative out-of-equilibrium "ratcheting" mechanisms must be implemented during the system's operation, in order to make use of the mechanical work generated before the material returns to its starting state.

The present Review aims at providing physical and chemical strategies to incorporate ratcheting mechanisms in the emerging domain of mechanically active molecular materials (Box 1, Figure I, Key Figure). In a first section, we will survey the diversity of stimuli-responsive materials that have been designed to generate mechanical motion, since all of them could be potentially ratcheted. In a second part, we will highlight recent advances in the design of ratcheting strategies at both the macroscopic and molecular scales in order to extract and 
(B)
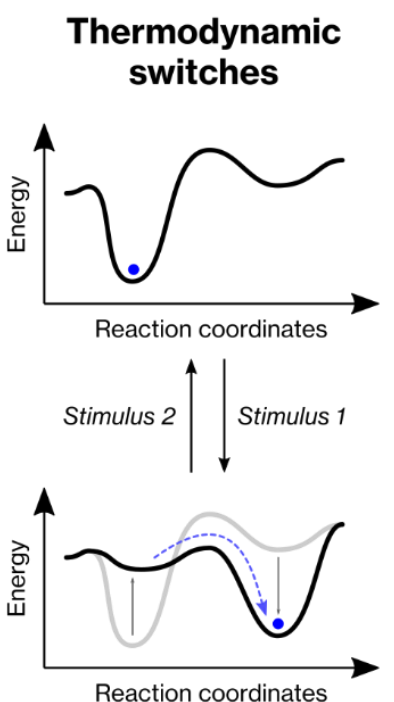

(C)

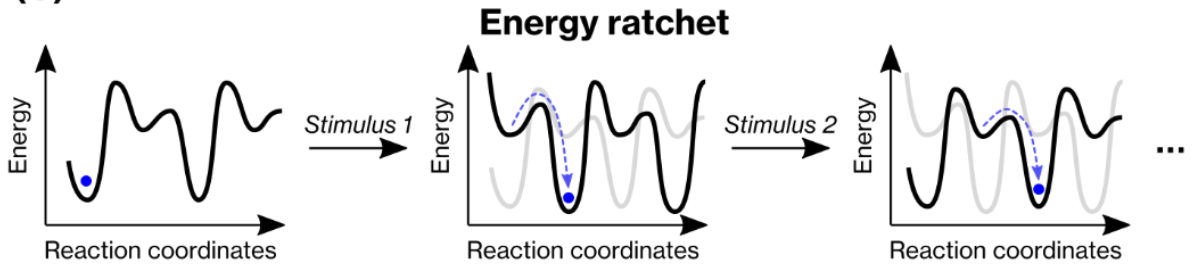

Information ratchet

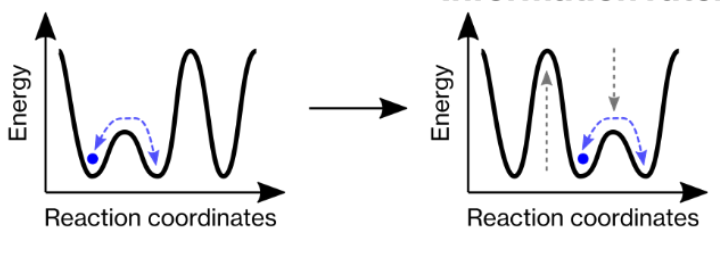

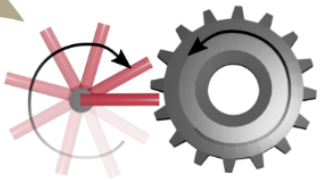

Ratchet

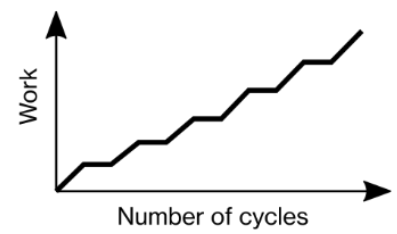

Number of cycles

\section{Metastable (photo)switches}

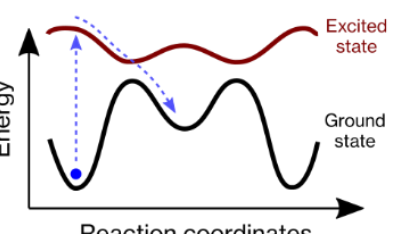

Light $\uparrow \downarrow$ Light

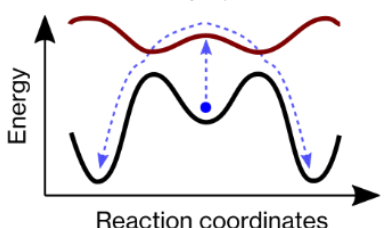

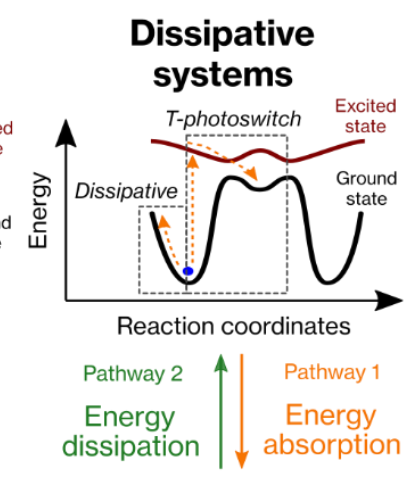
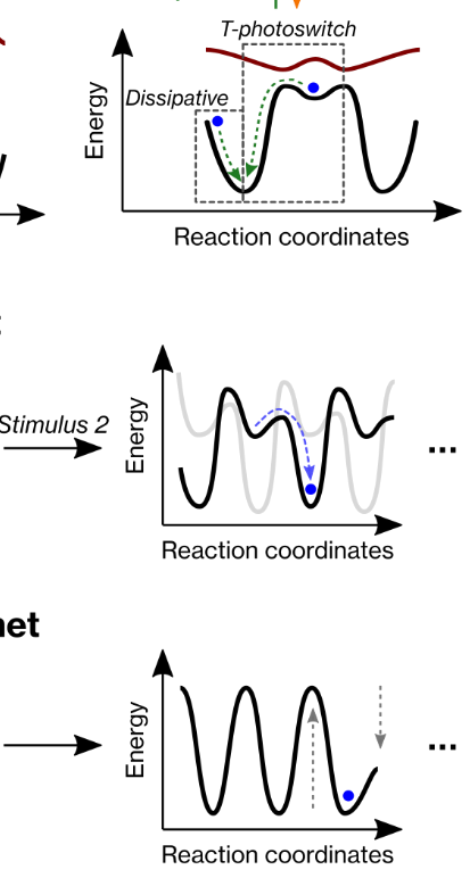
Upon appropriate stimuli, molecular switches can reversibly evolve between different states which correspond to local minima of their energy profiles. In general, the switching process takes place according to two different mechanisms. Either modification of the ground state energy of the molecule leads to a different stable state, or the switch reaches a metastable state of higher energy when supplied with energy.

In the first case, application of a stimulus changes the energetic profile of the molecule. Consequently, the initial state is not the most stable one anymore and the system thermally relaxes to its new thermodynamic equilibrium, whence the term 'thermodynamic switch' (Figure IB, left). The relative populations of all states are governed by Boltzmann distributions. Another stimulus can either revert the molecule back to its initial state or lead to another new thermodynamic equilibrium.

The second scenario involves the absorption of energy to reach a metastable state. Photoswitches follow this mechanism; those molecules absorb photons to reach an excited state and relax to a metastable state of their ground energy, whence the term 'metastable switch'. Since this conversion is not governed by thermal processes, the relative populations of the different states do not follow Boltzmann distributions. From its metastable state, the switch can reabsorb a photon to go back to its stable ground state through the excited state. Therefore, by tuning the energetic barrier between the states and working with appropriate wavelengths, one can reach long half-lives of the metastable configuration and actuate the molecule between these two states (Figure IB, middle).

A third scenario involves so-called dissipative systems which are constantly being driven out-of-equilibrium by absorbing energy and which are continuously dissipating this energy to their surrounding when relaxing to their thermodynamically stable state (Figure IB, right). The out-of-equilibrium state cannot be maintained without energy supply. Photoswitches with short-lived metastable states are dissipative since the absorption of light energy breaks microscopic reversibility when the switch concomitantly relaxes back to its global equilibrium through a low activation barrier of the ground state. Molecular motors are also dissipative systems, but where the energy dissipation preferentially occurs in one direction by implementing ratcheting strategies. For instance, dissipative photoswitches change their geometry during their operation but the molecular motion averages to zero over time; photodriven molecular motors, on the other hand, continuously move in one direction and their motion can therefore be exploited at the nanoscopic scale to produce an increasing work on 
their environment. Ratcheting strategies can be of different types and involve either $i$ ) "energy ratchets" that have no reference to the position of the particle and in which biased potentialenergy minima and maxima are repeatedly modified (Figure IC, top), or ii) "information ratchets" which rely on modifying the height of an energy barrier depending on the position of the particle on the potential-energy surface (Figure IC, bottom).

\section{Macroscopic actuation from stimuli-responsive molecular materials}

Molecular switches may trigger various changes in chemical and physical properties at the nanoscale upon appropriate stimulation, and these changes may be in turn amplified - by diverse processes - up to the generation of macroscopic actuators.[6] As discussed hereafter, such materials can be synthesized from switches involving thermodynamic, metastable, or dissipative states (Box 1), and therefore they can differ by the way they use (i.e. absorb and release) an external source of energy to convert it in molecular motion.

\section{Materials based on thermodynamic switches}

The functioning principle of a thermodynamic switch relies on the application of an external stimulus to alter its ground state energy profile and to shift its thermodynamic equilibrium (Box 1 and Figure IB, left). The mechanical motion generated by such switches arises from their reversible thermal relaxation between an initial state (becoming thermodynamically unstable) and a final state (becoming thermodynamically stable). While chemoresponsive switches reach a new stable state upon changes in their chemical environment (such as pH or metal ions concentration), thermoresponsive switches are based on phase transitions leading to different temperature-dependent equilibria.

Several strategies have been used to design chemoresponsive (macro)molecular actuators. For instance, hydrogels based on $\mathrm{pH}$-responsive polymers have different swelling equilibria depending on protonation (or deprotonation) of basic (or acidic) side groups. P(HEMA-co-AA) (poly[(2-hydroxyethyl methacrylate)-co-(acrylic acid)) and P(HEMA-co-DMAEMA) (poly[(2hydroxyethyl methacrylate)-co-(2-(dimethylamino)ethyl methacrylate) were used to create a microfluidic valve that could sort aqueous solutions depending on their $\mathrm{pH}$, exploiting the opposite swelling behavior of these two polymers under basic and acidic conditions [7]. Another strategy consists in using responsive supramolecular units in the system; for instance by involving DNA sequences which are known to be sensitive to various stimuli $[8,9]$. In particular, i-motifs $[10,11]$, which are formed under slightly acidic $\mathrm{pH}$, have been used to 
design DNA-based bilayered actuators [12]. Upon acidification of the medium, cytosine-rich DNA sequences form these i-motifs, which act as new physical crosslinks in the hydrogel, therefore inducing a stress in one layer which bends the entire material. Using more complex actuating units based on molecular machines using mechanical bonds, $[13,14]$ our group developed polymerizable [c2]daisy chains to form a contractile gel sensitive to $\mathrm{pH}$ changes (Figure 1A) $[15,16]$. In basic conditions, the macrocycles preferentially bind the triazolium stations and the molecule stands in a contracted state; in acidic conditions, however, the secondary amines are protonated and the macrocycles preferentially bind the dialkylammonium stations, resulting in an extended conformation. At the molecular scale, this sliding motion occurs over approximately $1 \mathrm{~nm}$ but, when the [c2]daisy chains are polymerized into a covalent network by copper-catalysed Huisgen[3+2] cycloaddition, the nanometric displacements are correlated through space and are amplified up to the macroscopic scale. The [c2]daisy-chain-based gels contract to approximately $60 \%$ of their initial volume in basic conditions and recover their initial shape in acidic conditions. Metal ions can also trigger actuation in responsive materials. For instance, guanine-rich DNA sequences are sensitive to monocationic ions, such as potassium or sodium, leading to the formation of G-quadruplexes. Following a mechanism similar to the one described for materials based on i-motifs (vide supra), bilayered actuators bending upon addition of potassium ions were designed (Figure 1B) [12]. Alternatively, DNA-DNA duplexes with cytosine-cytosine mismatches, which are sensitive to silver ions, were used to fabricate metal-sensitive hydrogel actuators [17]. Supramolecular redox processes have also been considered as stimuli for actuators. In an early example, Harada et al. developed a hydrogel actuator based on the redox sensitive ferrocenecyclodextrin interaction. In reductive conditions, these two molecules form an inclusion complex in water because of the hydrophobicity of ferrocene. Under oxidative conditions, however, the ferrocene switches to its cationic ferrocenium ion and electrostatic repulsion breaks the complex with cyclodextrin. In the hydrogel, oxidation induces a reduction of the crosslinking density due to the dissociation of inclusion complexes, which results in an expansion of the material (Figure 1C) [18]. Recently, a similar material based on viologencyclodextrin interactions was reported as redox responsive actuator. In that case, inclusion complexes consisting of cyclodextrin and dimers made of reduced radical-monocationic viologen act as additional reticulating units which break upon oxidation, leading to an expansion of the material [19]. Alternatively, oligoviologen units inserted into a polymeric 
hydrogel can act by themselves as redox-responsive units. Reduction of the viologens leads to the formation of complexes between the reduced radical-cationic species inducing the folding of the polymer chains and the subsequent material's contraction [20].

\section{Thermodynamic switches}

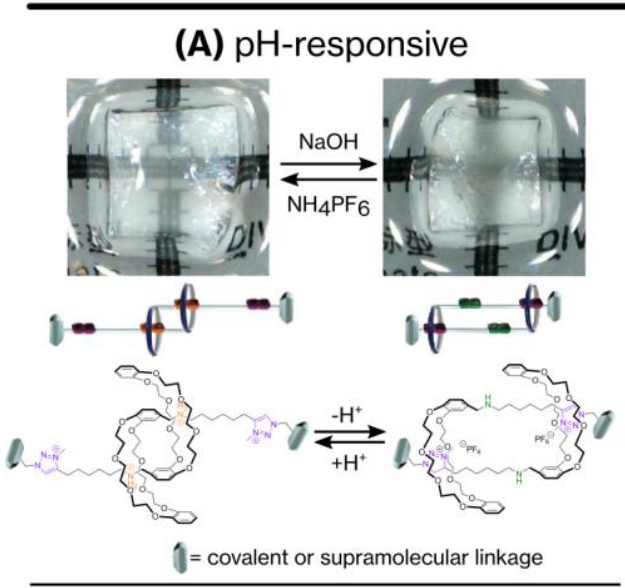

(C) Redox-responsive
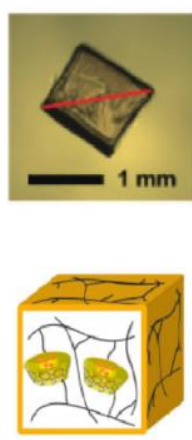

(B) Responsive to metal ions

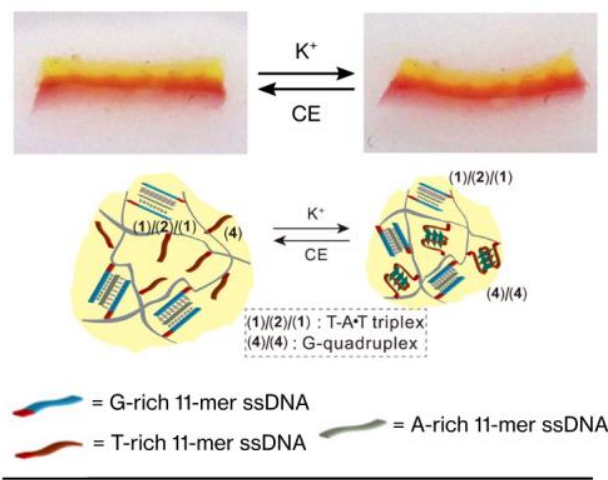

(D) Thermoresponsive
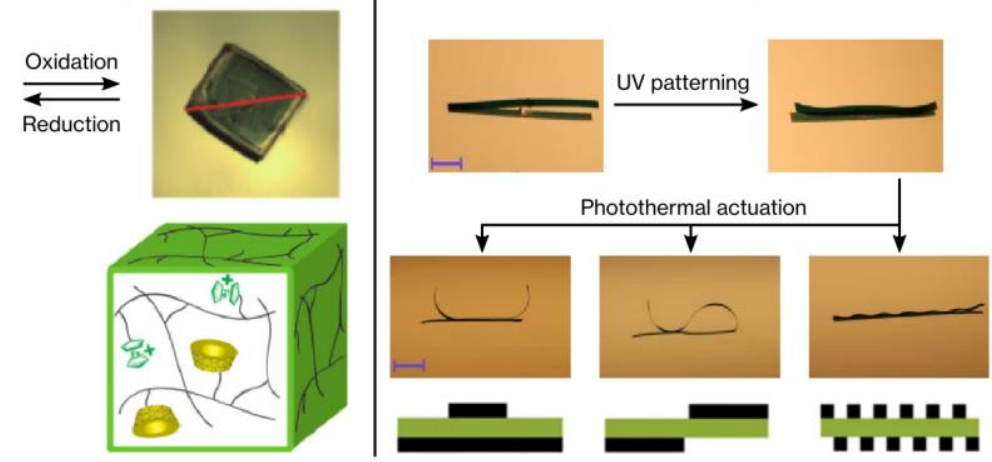

Figure 1. Representative examples of actuators based on thermodynamic switches. (A) $\mathrm{pH}$ responsive chemical gel based on [c2]daisy chains. Under basic conditions, the macrocycles preferentially bind the triazolium stations (purple) and the switch is in a contracted conformation. In acidic conditions, the secondary amines (green) are protonated and the macrocycles preferentially bind the dialkylammonium stations (orange), resulting in an extended state of the [c2]daisy chains. The molecular motion is amplified up to the macroscopic scale in the chemical gel. Black ticks in the snapshots are separated by $1 \mathrm{~mm}$. (B) DNA-based actuator responsive to metal ions and schematic representation of the yellow layer. The red layer is mainly composed of PNIPAM, while the yellow layer is made of polyacrylamide. Upon addition of potassium ions, the yellow layer can form G-quadruplexes which act as new crosslinks, thus leading to a contraction of this layer and, hence, a bending of the material. CE stands for crown ether. The length of the gels is $\sim 2 \mathrm{~cm}$. (C) Redox-responsive actuator based on ferrocene-cyclodextrin complexes. In reductive conditions, ferrocene forms inclusion complexes with cyclodextrin because of its hydrophobicity; in oxidative conditions, the formation of the ferrocenium cation leads to dissociation. As the crosslinking density decreases, expansion of the gel occurs. (D) Reprogrammable thermoresponsive LCN actuator. An initial reversible photopatterning step, leading to a minimal deformation of the material, creates stress distributions in the network. Heating the material with light via photothermal agents actuates the material following these patterns (representation below). Scale bars: 5 mm. Figure 1A: from 10.1021/jacs.7b06710, Figures 1 and 3. 
Figure 1B: from 10.1021/jacs.6b10458, Figure 3. Figure 1C: from 10.1002/anie.201300862, Figure 3. Figure 1D: from 10.1038/s41467-018-06647-7, Figure 4. The four of them have been adapted.

Different strategies have also been proposed in order to reach thermoresponsive actuating devices. One of them relies on the use of common thermoresponsive polymers such as PNIPAM (poly(N-isopropylacrylamide)) which displays a LCST around $32^{\circ} \mathrm{C}$ in water, meaning that its solubility substantially decreases above this temperature. As a result, PNIPAM-based hydrogels shrink when heated. Recent reports involving PNIPAM include the design of bilayer composites exhibiting bending motion [12,21,22], and of robotic devices such as grippers that could reversibly grab and release an object [23]. Liquid crystalline networks (LCNs) are also common thermoresponsive materials where the phase transition between the ordered, liquid-crystalline phase and the disordered isotropic phase leads to volume changes. Since light can be precisely addressed spatially and temporally, LCNs are commonly doped with photothermal agents [24] such as carbon nanotubes [25], gold nanoparticles [26,27] or organic dyes [28]. While most designs result in bistable actuators, recent reports have shown reprogrammable systems where different final states can be reached. For instance, Lahikainen and colleagues reported an azobenzene-based LCN that could be reversibly patterned with UV light through photomasks. As a consequence, different stress distributions were achieved inside the material, leading to different deformation modes once the material was heated via photothermal effect (Figure 1D) [29]. Recently, incorporation of diarylethene units inside these LCNs allowed actuation only when both UV and visible light were applied, thereby acting as an AND logic gate [30]. Finally, Zeng and coworkers reported so-called 'Pavlovian' materials based on LCNs [31] where the material, initially inert to light, could be 'trained' to become light-sensitive by allowing the diffusion of photothermal agents inside the material during simultaneous heat/light stimulation [32]. Interestingly, DNA-based materials have also been reported as thermoresponsive actuators. The dissociation of DNA duplexes could be controlled by heating the material with light using gold nanoparticles or nanorods as photothermal agents, leading to a change in crosslinking density which triggered actuation [33]. The reversibility of the DNA complexes formation in the absence of light allowed the recovery of the initial shape.

Oscillating phenomena have also been exploited to trigger back and forth actuation using either chemoresponsive or thermoresponsive systems. This approach has the advantage of 
cyclically modulate the global energy profile of a molecular material over time without the need of sequential inputs. Most oscillating chemoresponsive materials are based on the Belousov-Zhabotinsky (BZ) reaction. For instance, the redox-responsive catalyst of the BZ reaction can be covalently incorporated inside PNIPAM-based hydrogels. The oscillating change in hydrophilicity of the catalyst result in an oscillating modulation of the hydration equilibrium of the polymer, thereby leading to an oscillating volume change of the material (Figure 2A) [34,35]. An oil pump was recently reported with this type of material [36]. Other oscillating reactions have also been used to design time-dependent actuating materials [37], such as an enzymatic oscillating reaction powered by ATP that led to an oscillating concentration in calcium ions. These ions could reversibly bind to phosphate groups along the polymer chains of an hydrogel, thereby modulating the crosslinking density and thus inducing periodic volume changes [38]. Oscillating materials can also be designed from thermoresponsive materials. The groups of Broer and Meijer thoroughly investigated this phenomenon with thermotropic LCNs doped with photothermal agents. In this case, irradiation of the material induces its bending; the bent part, however, blocks the light source from irradiating the material. It therefore relaxes back thermally to its initial position, allowing the light source to irradiate again the LCN, which bends again. This self-shadowing effect leads to a sustained oscillations of the polymer films (Figure 2B) [39-41]. Finally, such a kind of device can be coupled to another material collecting and transducing the generated mechanical motion. This was reported, for instance, with oscillating materials driven by photothermal effect (vide supra) and coupled to piezoelectric PVDF (poly(vinylidene fluoride)) films [42]. The observed piezoelectric effect arises from the oscillation of the PVDF film, which comes from the light-induced oscillatory motion of an LCN material. Consequently, the oscillating motion of the LCN is transduced, via the PVDF, as an oscillating potential difference between two electrodes. It is worth noting that this potential difference averages to zero over time, highlighting that, overall, no continuous work is extracted (Figure 2C).

Although thermodynamic switches allow the design of a wide variety of materials, they are often associated with several drawbacks. Chemoresponsive systems usually suffer from substantial fatigue after a few operating cycles because of the accumulation of chemical waste over time. Thermoresponsive materials, on the other hand, cannot be directly addressed very precisely with temperature, thus requiring very often the use of photothermal agents, since 
light can be more easily focused on precise spots at precise times. In the following section, we will see how light can be used to actuate materials following totally different energy profiles.

\section{Oscillating materials}

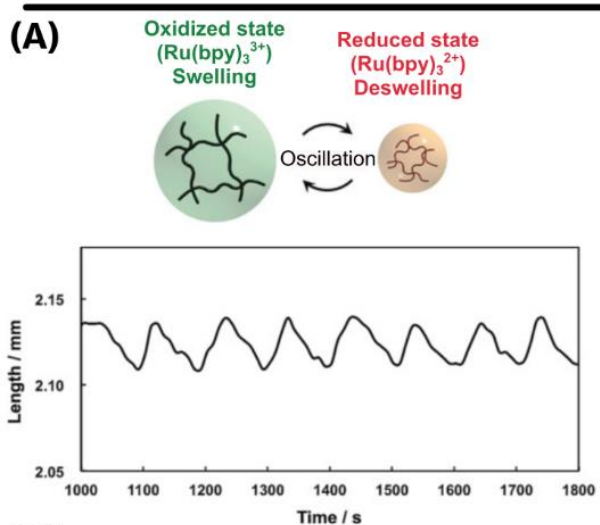

(C)

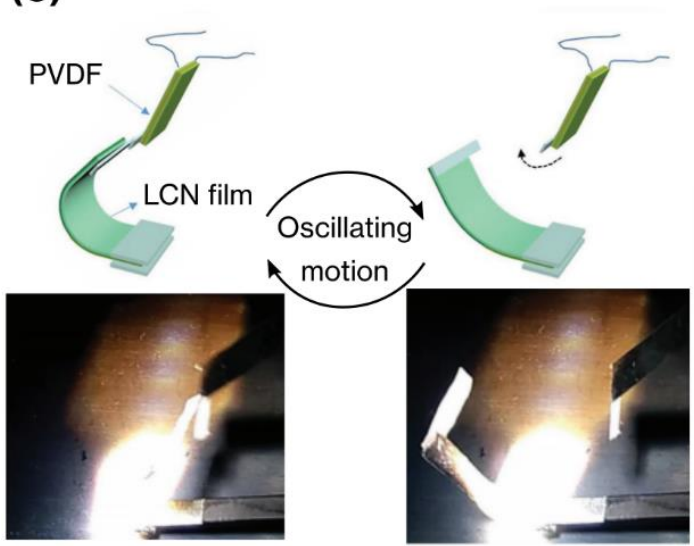

(B)
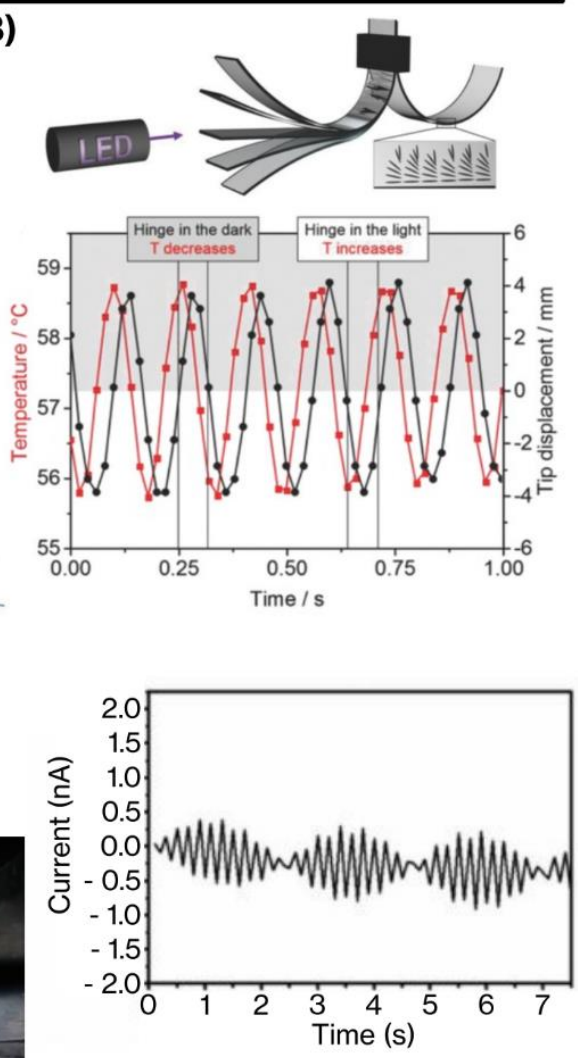

Figure 2. Representative examples of oscillating materials. (A) Microgels based on PNIPAM were functionalized with tris(bipyridine)ruthenium $\left(\mathrm{Ru}(\mathrm{bpy})_{3}\right)$. Since it is swollen in a BZ reaction medium, the oxidation state of $\mathrm{Ru}(\mathrm{bpy})_{3}$ cyclically changes over time and, hence, modifies the hydration equilibrium of the polymer gel (top). Consequently, the microgels cyclically swell and shrink over time (bottom). (B) LCNs with a splay alignment were doped with photothermal agents (top). Upon local irradiation, the photothermal effect from the dopants induces local heating of the material and, therefore, its bending. Consequently, the tip of the LCN films blocks the light beam from reaching its irradiation point; rapid heat dissipation triggers the unbending of the material to its initial state and the cycle is repeated (bottom). (C) Oscillating LCN films were used to modulate the potential difference between two electrodes. The oscillating motion of the light-sensitive was used to hit a PVDF film and induce its own mechanical oscillation (left). The oscillating deformation of the PVDF creates an oscillating potential difference between two electrodes (right). The 'beating' aspect is caused by a mismatch between the oscillation frequency of the LCN and the oscillation frequency of the PVDF (due to its intrinsic elasticity). Importantly, the potential difference averages to zero over time. Figure 2A; from 10.1002/anie.201809413 Figures 1 \& 5. 2B: from 10.1002/adma.201606712, Figure 2. 2C: from 10.1002/adom.201800131, Figure 6. 


\section{Materials based on metastable switches}

Under light irradiation, photoswitches can reach a metastable state of higher energy (Box 1 and Figure IB middle), thus leading to a state probability inaccessible for thermodynamic switches following Boltzmann distribution. This excitation process is photodynamic as it is reversible through the excited state between the metastable and stable ground states. In addition, this reversibility can be complemented by a thermal process going from the metastable state to the stable one when the energy barrier of the ground state is low enough to be crossed, thus leading to dissipative systems which can be maintained out-of-equilibrium under light irradiation (Box 1 and Figure IB, right). Note that such photoswitching systems do not follow the principle of microscopic reversibility, and as it is the case for switches purely actuating back and forth along their ground states such as chemical systems displaying detailed balance. Consequently, an actuating material based on photoswitches can reach, depending of the height of the ground state energy barrier, either a metastable shape that can be reset by photoexcitation, or an out-of-equilibrium shape that eventually relaxes back thermally.[43] Several molecular photoswitches have been recently implemented to access actuating macroscopic materials $[44,45]$.

Azobenzene derivatives are probably the most used ones. Upon light irradiation, the stable trans-isomer is isomerized to its metastable cis-form. This process is reversible either by thermal relaxation or by light irradiation [46]. Trans-azobenzene, with an elongated shape, has been widely used as dopant in LCNs which can then actuate according to either a photothermal or a photomechanical mechanism. On one hand, since azobenzene absorbs light, it can be used as a photothermal agent to trigger the actuation of thermoresponsive LCNs by the photothermal effect. On the other hand, the photomechanical effect, which arises from the bent structure of the cis- isomer, can induce destabilization of the liquid crystalline phase leading to disorder which results in a volume change of the material. However, the relative contribution of each mechanism is still a subject of investigations [47]. LCNs have great potential to be used in soft robotics because of their mechanical properties and their efficient stimuli-responsiveness; therefore, doping them with light-sensitive molecules paves the way towards remotely controlled soft robotics systems.[48,49] While most azobenzene-based LCNs lead to materials which can actuate between two states [50], recent reports presented reprogrammable materials including for instance liquid crystalline elastomers [51] or LCN/thermoplastic composite materials [52]. In this last example, the presence of a 
thermoplastic polymer allows for shape molding and reprogramming at a temperature close to the glass transition temperature while light is used for actuating the LCN (Figure 3A) [52]. Recent developments also include the design of more complex materials that are multi-stimuli responsive $[53,54]$ or that can get crossed-information from the environment to trigger their actuation [55]. Azobenzenes in their trans- form are also known to form inclusion complexes with cyclodextrins [56]. Upon UV light irradiation, the formation of the cis-isomer leads to the dissociation of the complex. This unthreading process that has been exploited in chemical gels based on [c2]daisy chains [57] and [2] rotaxanes [58] leads to a volume change of the material upon light irradiation.

Spiropyran has also been widely used for the design of photoactuated materials $[6,59]$. Under light irradiation, it can reversibly isomerize between its closed spiropyran form and its open merocyanine form. The stimuli-responsiveness of spiropyran-based materials originates from the difference in polarity between these two isomers. In water, in the absence of any charged substituents, the zwitterionic merocyanine is the most stable isomer and its ring closure under light irradiation leads to the neutral spiropyran, which is less solvated by water. When incorporated in a hydrogel, this change in solubility induces a shrinkage of the material upon light irradiation, subsequently leading to a bending towards the light source [60]. This tendency can be reversed if the spiropyran is functionalized with negatively charged sulfonate groups which increase the net charge of the molecule in its closed form (Figure 3B) [61]. As for azobenzene, new materials with improved performance are still regularly reported. For instance, the group of Stupp designed a hybrid hydrogel made of supramolecular nanofibers that are chemically cross-linked to a photo-responsive chemical network containing spiropyran side chains [62]. As a gradient of hydrophobicity occurs upon light irradiation, a sixarm flower-shaped hydrogel was bent in different configurations by precisely addressing the light on each petal. 


\section{Metastable switches}

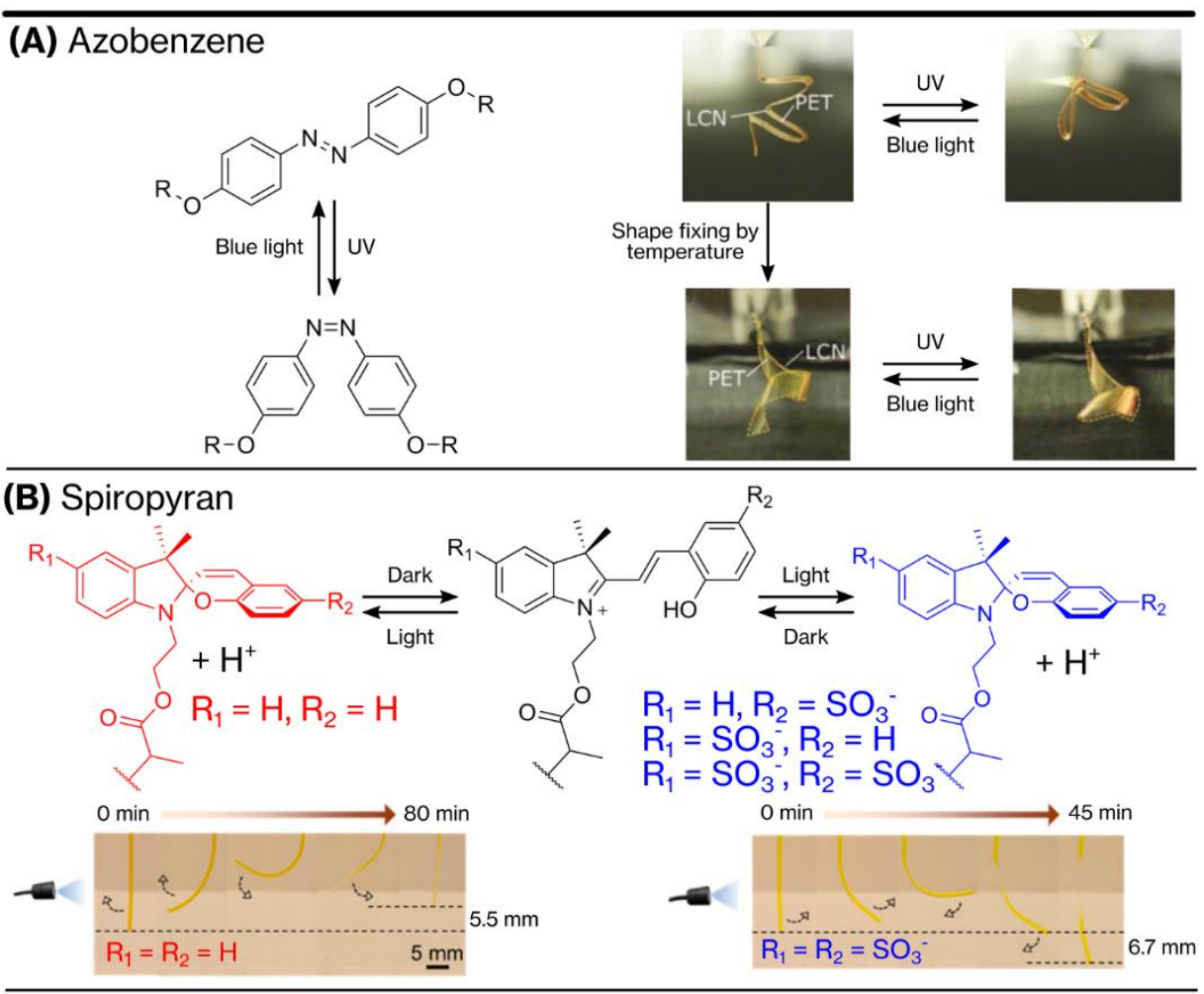

(C) Diarylethene
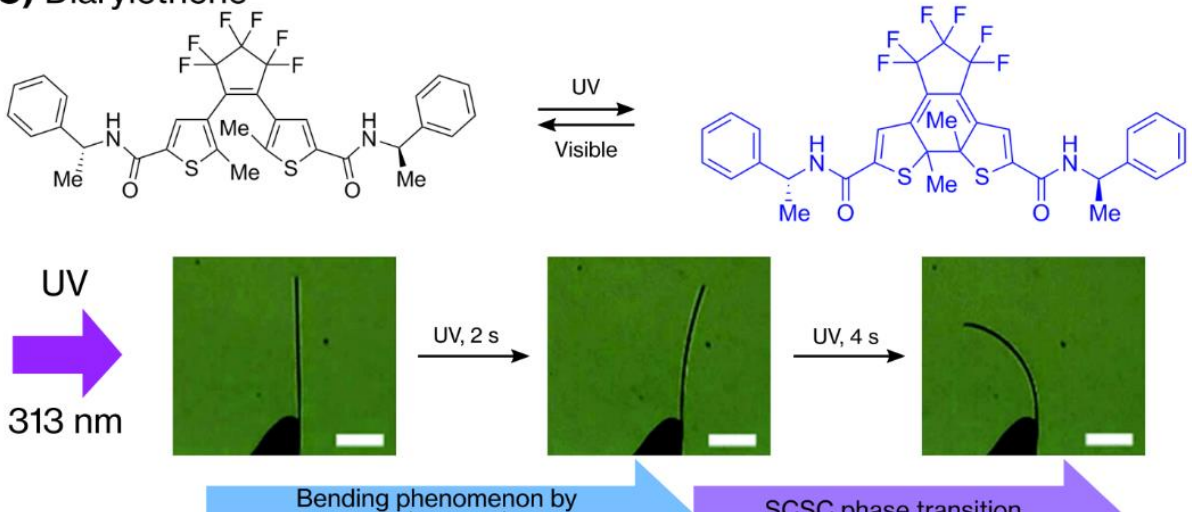
crystal expansion

Figure 3. Representative examples of actuators based on metastable switches. (A) Reprogrammable azobenzene-based LCN. The LCN is prepared on a thermoplastic substrate (PET, poly(ethylene terephthalate)) that can be deformed when heated above its glass transition temperature. Different modes of actuation under UV light can therefore be achieved with a single material. The length of the material is $2 \mathrm{~cm}$. (B) Spiropyran-based gels. When merocyanine has no sulfonate group, ring closure under UV decreases the net charge of the molecule, leading to a contraction towards the light source. Yet, when merocyanine has sulfonate groups, ring closure under UV increases the net charge of the molecule, leading to an expansion of the gel and, therefore, a bending motion away from the light source. In both cases, for prolonged irradiation times, unbending occur as the deformational gradient disappears. (C) Diarylethene-based crystal. The crystal is irradiated from the left side. Under UV, the crystal first bends away from the light source because of the change in geometry of the photoswitch which leads to an expansion of the crystal. For longer irradiation times, however, the higher population of photocyclized molecules triggers a single-crystal-to-single-crystal transition with smaller final lattice 
parameters, leading to a bending motion towards the light source. Scale bars: $250 \mu \mathrm{m}$. Figure 3A: from 10.1002/anie.201915147, Figure 4. Figure 3B: from 10.1021/jacs.0c02201, Figure 4. Figure 3C: from 10.1039/DOSC05388K, Figure 1.

Diarylethenes can also be used to generate mechanical work because light irradiation triggers the electrocyclization of the molecule. When organized in crystalline lattices, this change in geometry can be translated up to the macroscopic scale by reorganization of the irradiated crystalline phase. Since the first report in 2007 [63], many more systems have been described (Figure 3C) [64-66].

In the first part of this Review, we have discussed different mechanisms that can be used to generate mechanical actuation from molecular materials. However, in most cases, the work performed during the actuation comes undone when the material returns to its initial state. In order to extract increasing amount of work from such materials and to use repeatedly the mechanical work produced to perform a task, one should enforce them to undergo unidirectional motions by "ratcheting" their actuations.

\section{Extracting continuous mechanical work from molecular systems and materials by implementing ratchet mechanisms}

To produce a continuously increasing mechanical work on its environment, an actuator must involve a symmetry-breaking process, as the system must follow a distinct trajectory when returning back to its initial stable state (Box 1 and Figure IA, right). Symmetry breaking of actuating molecular materials can take place by implementing different types of ratchets acting at different length scales. They can for instance $(i)$ take advantage of an asymmetric external stimulation; (ii) involve a built-in asymmetric response of the material, or (iii) rest on the design of a ratchet mechanism at the nanoscale in order to upgrade molecular switches in molecular motors (Box 1 and Figure IC). $[67,68]$

Asymmetric interaction with the environment. One possibility to achieve unidirectional motion of an actuating material is to make it interacting with a ratcheted surface, as already demonstrated with spiropyran-based gels $[69,70]$. Oriented translational motion as well as rotation over several cycles was reported for such materials [62]. A bent gel was also cyclically swollen and shrunk with light on an asymmetric ratcheted surface (Figure 4A). The dissymmetric geometry of this serrated surface precluded motion to the right, thus forcing the material to repeatedly move in the same left direction under sequential light irradiation 
[69]. An alternative strategy consists in an asymmetric stimulation of the actuating system. Early reports show that either simultaneous or alternate UV and visible light irradiation could induce the rolling motion of a belt made of azobenzene-based LCNs [71] or of a gear using diarylethene-based crystals, respectively [72]. Recently, Pilz da Cunha et al. reported a soft robotic tetrapod with four LCN legs that could move and transport objects by selective and sequential irradiation of the legs (Figure 4B) [73]. Fischer and coworkers reported the translational motion of a tubular LCN caused by a wave-like propagation of a local expansion of the material. Light irradiation was locally applied progressively along the length of the network, biomimicking the motion of earthworms (Figure 4C).[74]

\section{Macroscopic ratchets}

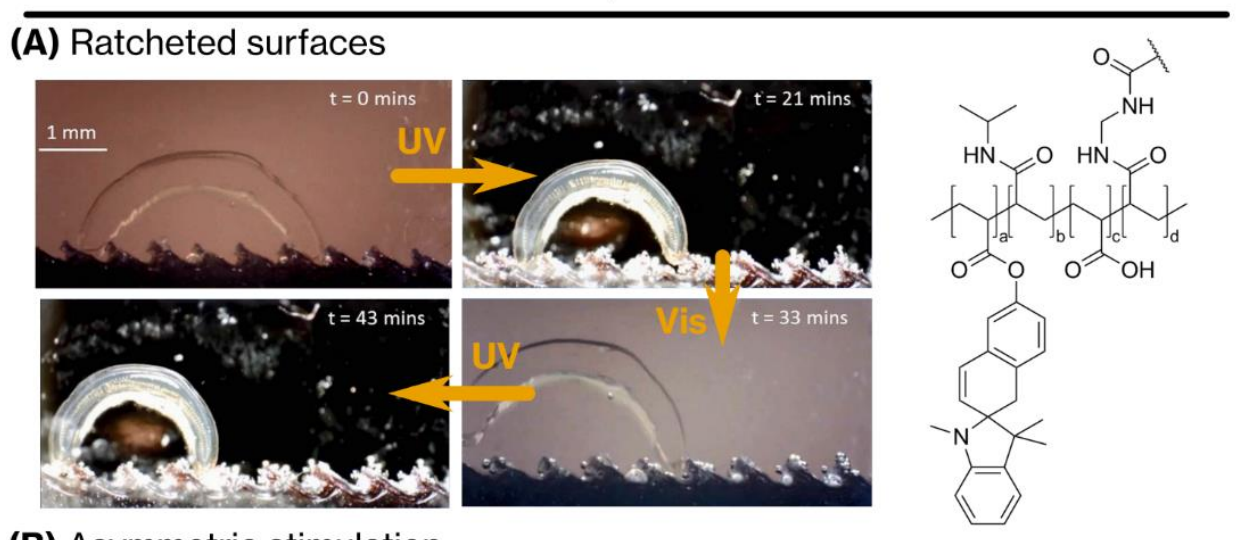

(B) Asymmetric stimulation

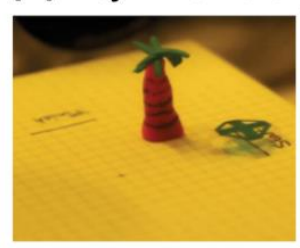

Initial state

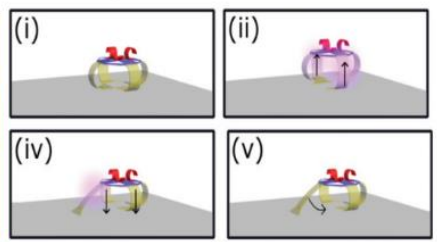

Light-driven motion
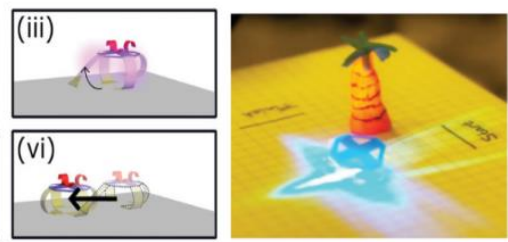

Final state

(C) Asymmetric stimulation

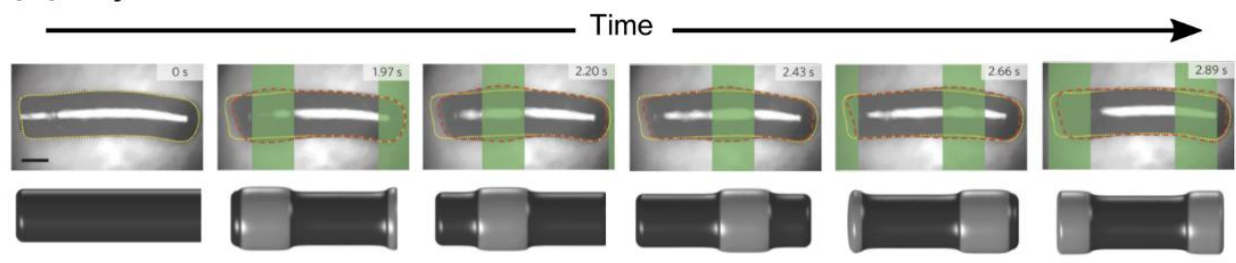

(D) Inherent asymmetry of the material
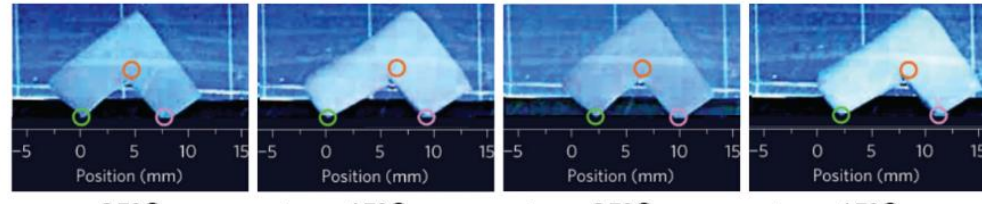

$25^{\circ} \mathrm{C}$
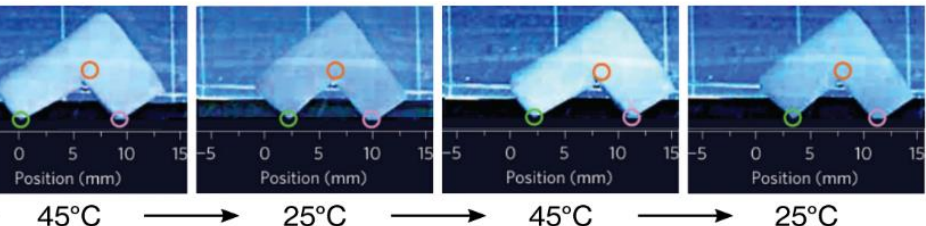

Figure 4. Design of macroscopic ratchets. (A) Spiropyran-based gel 'walking' on a ratcheted surface. The material itself is cycling between two shapes, while the asymmetry of the surface makes it move 
preferentially to the left. (B) Locomotion of a soft robotic device over more than $4 \mathrm{~mm}$ by asymmetric light stimulation according to the following mechanism: (i) a platform is mounted on four azobenzenebased LCN 'legs', (ii) irradiation of two opposite legs lifts the device while (iii) irradiation of a third leg with a tilted angle induces unfolding, (iv) stopping irradiation on the two opposite legs lowers the platform, (v) light removal from the third leg induces bending back to its initial shape, and (vi) a translating motion occurs as this bending motion drag the whole system. Snapshots on the left and right sides show the position of the platform before and after this sequence, respectively. (C) Snapshots of an LCN-based tubular material (top) with the corresponding schematic representation of the volume changes (bottom). Asymmetric light irradiation leads to the directional motion of the material. Local light irradiation (green areas) trigger volume expansion. The light beam travels along the length of the material, causing a wave-like propagation of the deformation, which in turn induces the translational motion of the tube, similar to the one of an earthworm. Scale bar: $200 \mu \mathrm{m}$. (D) Anisotropic PNIPAM-based gel containing precisely-oriented titanate nanosheets. The anisotropic swelling behavior drives the motion of the gel preferentially to the right side when the temperature is cycled above and below the LCST. Figure 4A: from 10.1016/j.snb.2017.05.005, Figure 5. Figure 4B: from 10.1002/advs.201902842, Figure 3. Figure 4C: 10.1038/nmat4569, Figure 2 Figure 4D: from 10.1038/nmat4363, Figure 5.

Inherent asymmetry of the macroscopic material. An alternative approach to extract mechanical work consists in using materials that are inherently asymmetric at macroscopic level. For instance, Kim and coworkers developed a PNIPAM-based gel containing macroscopically aligned titanate(IV) nanosheets, and which displays an anisotropic thermoresponsive swelling behavior. Interestingly, directional motion of a L-shape cut material occurred upon heating/cooling cycles, resulting from the anisotropic shape change which leads to an unidirectional translation of the centroid of the gel (Figure 4D) [75]. Microplatelets coated with azobenzene have been reported to perform continuous rotation under light irradiation when immersed in a liquid crystal. A feedback mechanism takes place between azobenzenes and mesogens at the interface of the object, modulating the effective light beam irradiating the material and, therefore, leading to an unidirectional rotation of the platelets [76]. Recently, photoresponsive micrometer-sized crystals capable of continuous, unidirectional twisting motion were reported. Light-induced $E \rightarrow Z$ isomerization of the allylidene malonitrile induced disruption of the crystalline phase of the material which resulted in anisotropic stresses leading to motion [77].

Ratcheting at the nanoscale: molecular motors. As already discussed, switches can reach several global or local minima of their energy profile depending on the stimulus applied (Box 1). A peculiar situation arises when the high energy state of a switch (pathway I) is continuously populated by energy absorption, and continuously depopulated by thermal 
energy transfer to its surrounding (pathway II) (Box 1 and Figure IB, right). Here, the resulting out-of-equilibrium situation can be ratchetted if the energy dissipation is enforced to occur in one preferential direction by molecular symmetry breaking, leading to a so-called molecular motor capable of unidirectional nanoactuation (Box 1, Figure 1C). Therefore, in contrast to mechanical switches which influence their surrounding environment as a function of their states, molecular motors move their subcomponents along a nonreversible energy profile, and influence their surrounding environment as a function of their trajectory. This confers them with the unique property to progressively and repeatedly increase the work they can perform on their environment. For instance, the group of Leigh reported the first chemically fueled molecular motor prepared from a [2]catenane [78]. Proper design of this interlocked structure allowed the unidirectional chemically powered rotation of one macrocycle with respect to the other by involving an information ratchet mechanism.[79] Nevertheless, most synthetic molecular motors are concomitantly photo- and thermally actuated with a ratcheting mechanism involving the presence of stereogenic centers.[80] The most common molecules of this type are overcrowded alkenes initially developed by the group of Feringa (Figure 5A) [81]. In such molecular motors, a first photochemical trans $\rightarrow$ cis isomerization takes place, leading to an unstable helical conformation which can relax to a stable one by thermal atropoisomerization, thus leading to a net $180^{\circ}$ rotation. This thermal helix inversion step is biased towards one particular direction due to the presence of an asymmetric carbon recovering its favored pseudo-axial conformation from an unfavored pseudo-axial one as obtained after light-irradiation. The repetition of these two steps completes a net unidirectional $360^{\circ}$ rotation. More recently, hemithioindigo motors have been developed, following the same mechanistic principles but rather being driven by visible light [81]. In these motors, the ratchet mechanism is encoded in the molecule itself. If such molecular motors are still not often exploited to extract mechanical work at higher length scales in materials, a number of innovative examples exploit the change in geometry at the photostationary state reached after irradiation. For instance, when overcrowded alkenes are used as chiral dopant in a liquid crystal, irradiation produces a change in the helicity of the motors, leading to the deformation of the cholesteric LC phase and subsequently inducing the rotation of a nanorod on the surface. Then, stopping the irradiation reverts the motion, cancelling the initial work produced through a reverse rotation of the nanorod [82]. The group of Feringa also reported supramolecular fibers containing amphiphilic overcrowded alkenes, where irradiation led to 

[83-85].

\section{Nanoscopic ratchets}

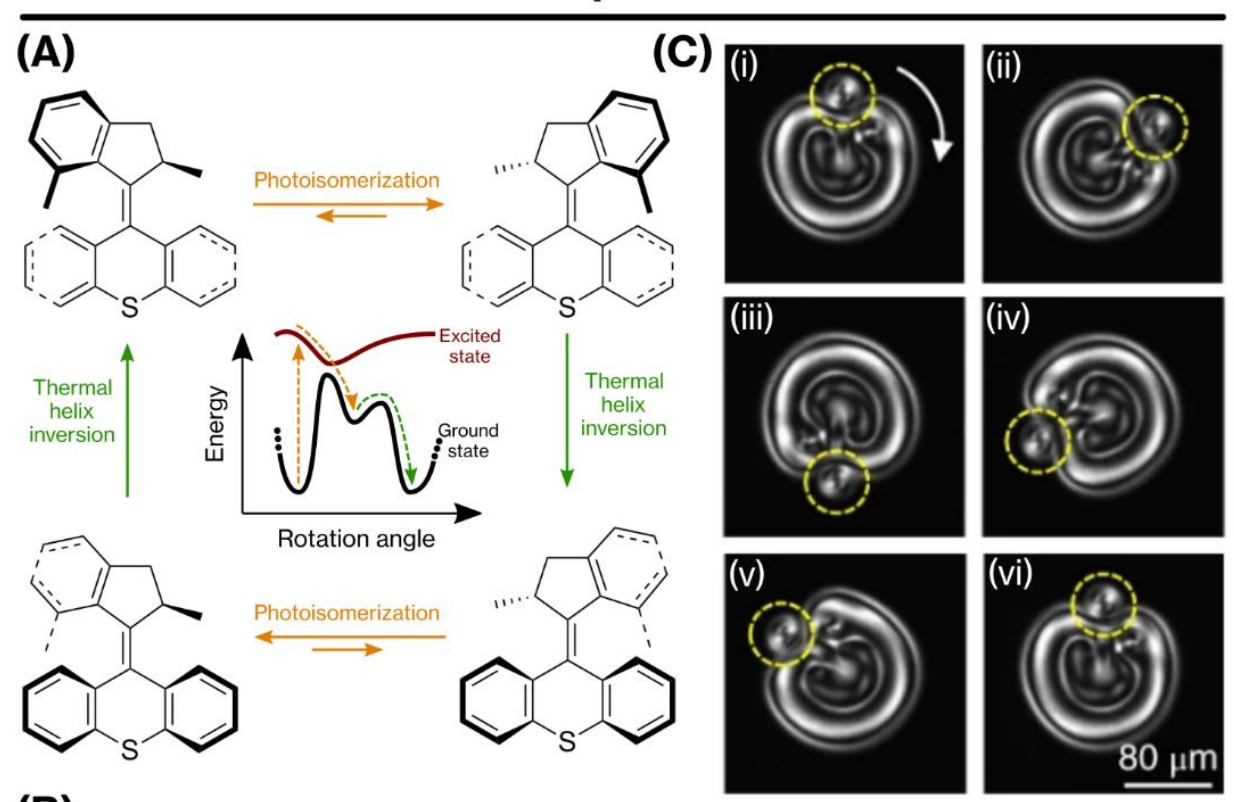

(B)

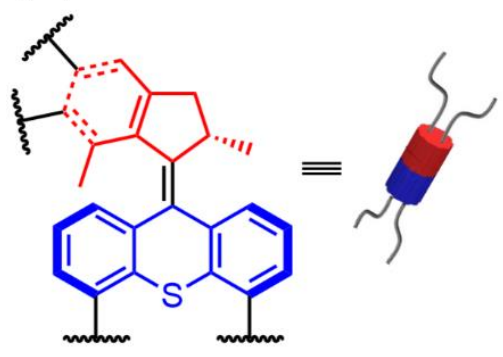

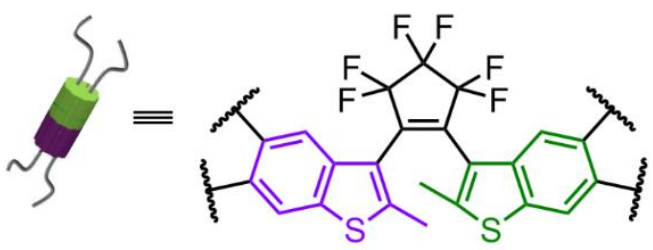
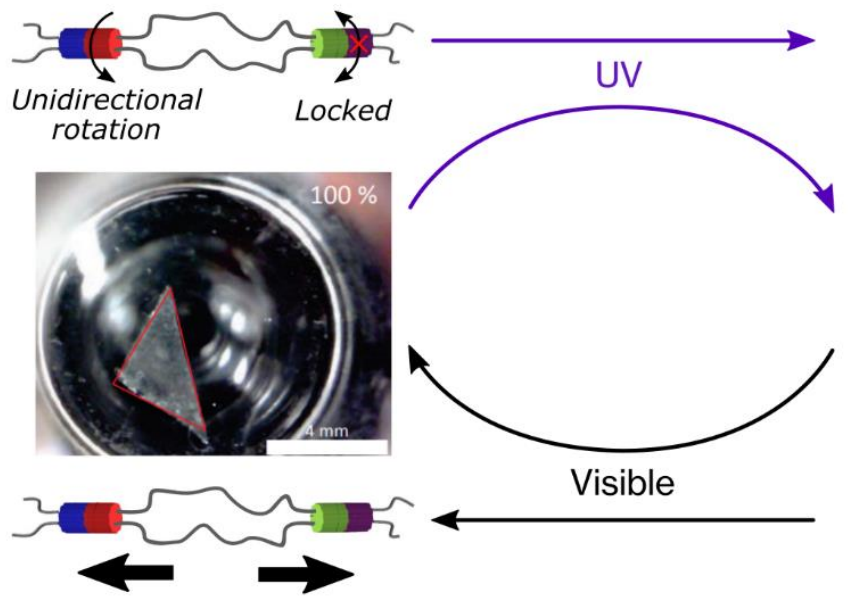

Visible
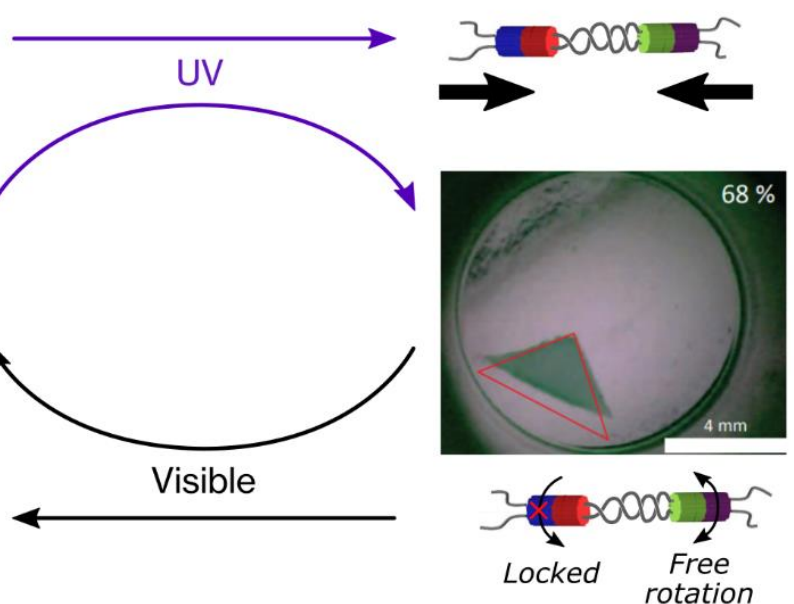

Figure 5. Design and application of molecular motors. (A) Mechanism for the unidirectional rotation of second-generation light-driven rotary motors based on overcrowded alkenes. It consists of two photochemical steps and two thermal isomerization steps. In the most stable configuration, the methyl group of the asymmetric carbon of the upper part has a pseudoaxial conformation. After the photoisomerization steps, the motor has an opposite helicity and, in this configuration, this methyl group has a less stable pseudoequatorial position. The most stable conformation is restored after 
thermal helix inversion. This ratcheted design of the molecule leads to unidirectional rotary motion. (B) Snapshots and schematic representation of chemical gels formed by rotary molecular motor crosslinks (red and blue cylinders) and diarylethene-based elastic releasers (purple and green cylinders). Upon UV irradiation, motors twist pairs polymer chains together, increasing the number of physical crosslinks and, hence, reducing the volume of the gel. The elastic energy stored in the material from the torque produced by the molecular motors can be restored upon visible light irradiation over 48 hours, as diarylethene units switch to their open form that can freely rotate and untwist the chains, recovering the initial volume of the gel. In other words, under UV, the work performed by the unidirectional motion of motors can be stored in the material because the diarylethenes are in their closed, 'locked' conformation. However, under visible light, the diarylethene modulators isomerize to their open form with free rotation around single bonds. Consequently, they will tend to rotate in the opposite direction to that of the motors to untwist the polymer chains. Overall, the process describes a continuous and unidirectional trajectory to be activated and reset. (C) Liquid-crystalline pattern continuously rotating under irradiation inside a molecular-motor-doped liquid crystal. The pattern is observed by polarized optical microscopy between crossed polarizers. The rotation of the pattern rises from a diffusion-driven feedback process occurring when the motor is isomerized. The torque produced can be used to transport a microscopic object (circled by yellow dotted line). Full rotation takes place in 23 minutes (from (i) to (vi)). Figure 5B: from 10.1038/nnano.2017.28, Figure 1 and Supplementary Figure 4. Figure 5C: from 10.1038/s41565-017-0059-x, Figure 5.

Our group reported on the formation of chemical gels incorporating such molecular motors as active crosslinks, and in which the work produced by each motor is directly stored as elastic energy in the network [86]. Indeed, because the rotation of the motors under UV irradiation induces a twisting of pairs of polymer chains, the increasing number of crosslinks from entanglements triggers a contraction of the material (through syneresis), together with an increase of its elastic modulus [87]. Interestingly, this elastic energy can be released by integrating light-triggered diarylethene moieties as additional crosslinking units, and through unwinding of the polymers chains. Because this unwinding take place at a different wavelength and in different locations of the materials, the entire system can be maintained out of thermodynamic equilibrium and ratcheted by the opposite directional rotation of the molecular motors (for instance turning clockwise) and molecular releasers (enforced to conversely turn anticlockwise) (Figure 5B) [88]. Therefore, the materials as a whole can contract and expand as a function of the trajectories and the frequencies of its molecular components and can be considered as intrinsically motorized. Using a different approach, Katsonis and Brasselet exploited the change in helicity of the molecule during the rotation of the motor, coupled to a diffusion-driven feedback mechanism, to create a continuously rotating pattern inside a liquid crystal under light irradiation. The torque produced was used 
to transport a cargo at microscale (Figure 5C) [89]. Irradiation of the motor leads to concentration gradients of its different states inside the liquid crystalline medium. In return, these concentrations gradients form different supramolecular structures between the motors and the liquid crystal that create a diffusion gradient inside the material. The interplay between concentration gradients and diffusion gradients leads to the formation of patterns that break their axial symmetry above a critical power, which then lead to the rotation of the pattern over time. While the unidirectional rotation of the motor is not directly responsible for the rotation of the liquid crystalline medium, it still highlights the potential application of the changes in helicity of the motor during its rotation cycle to design complex stimulisensitive supramolecular systems. These two last examples pave the way to the integration of molecular motors in globally dissipative systems in order to ratchet molecular materials at all scale.

\section{Concluding remarks and future perspectives}

The recent advances in the design and synthesis of mechanically active molecular materials are of both fundamental and practical interests. In particular, numerous progresses have been made by incorporating stimuli-responsive molecular units into polymer matrices. The various natures of these molecules permit to actuate materials using different sources of chemical or physical energy. As detailed above, the generated actuation can take place by several fundamentally distinct mechanisms leading to important thermodynamic consequences at all scales. One of the challenges in this approach is now to generate materials that can produce a continuous work on their environment, which requires the implementation of ratcheting strategies. With the recent development of ratcheting mechanisms at the nanoscale and the rise of molecular motors, new opportunities appear to exploit the work they can continuously produce at nanoscale by its amplification in macroscopic materials. A few examples have already shown that the ratchetted actuation of molecular motors can be preserved in properly designed artificial materials, but basically everything remains to be explored in this new kind of integrated motorized molecular systems. In particular, the rationalization of the ratcheting amplification from nano- to macroscale should be understood and generalized. Their full potential in terms of non-classical actuation and efficiency should also be clarified in comparison with simpler switching systems. In this direction, a number of stimulating 
challenges are certainly facing synthetic chemists and their colleagues at the interfaces with physics and engineering for the years to come (see Outstanding Questions).

\section{Glossary}

[2]catenane: mechanically interlocked structure composed of two intertwined macrocycles. The term is derived from the Latin for "chain" (catena).

[2]rotaxane: mechanically interlocked structure composed of dumbbell shaped molecule threaded through a macrocycle. The term is derived from the Latin for "wheel" (rota) and "axle" (axis).

Active material: material that can perform a task by energy dissipation from its environment. In particular, when such materials can sense and adapt to what happens in their environment, when they display motility, or if they reorganize themselves to perform multiple tasks, they are sometimes named as "life-like" materials.

BZ reaction: Belousov-Zhabotinsky reaction; an oscillating redox reaction taking place in acidic aqueous solution in the presence of bromine derivatives, transition metal catalysts and reducing agents, and driven by non-linear thermodynamics.

[c2]daisy chain: mechanically interlocked rotaxane composed of two identical molecules consisting of a ring (macrocycle) covalently linked to an axle. The axle of one molecule is threaded through the macrocycle of the other one, thereby leading to a cyclic topology [c2].

Detailed balance: principle stating that, at equilibrium, every process occurs at the same rate than its reverse process. This principle is therefore tightly related to microscopic reversibility.

LCN: Liquid Crystalline Network; a polymer network composed of mesogenic units. The phase transition from the ordered mesophase to the disordered isotropic phase leads to volume expansion when the material is heated. Cooling the material back to its liquid crystalline phase allows the recovery of its initial shape.

LCST: Lower Critical Solution Temperature; critical temperature under which a polymer is fully miscible in a solvent, for any composition. Conversely, the UCST (Upper Critical Solution Temperature) is the critical temperature above which a polymer is fully miscible in a solvent, for any composition. Crossing these critical temperatures can lead to macroscopic shrinkage/expansion of the polymer materials. 
Mechanical bond: entanglement in space between two or several molecular components and that cannot be undone without breaking a covalent bond. Among the possible mechanically interlocked structures, there are catenanes and rotaxanes, for instance.

Microscopic reversibility: principle stating that, the probability of any trajectory of a microscopic process through phase space equals that of the time reversed trajectory. It is tightly related to detailed balance for chemical reactions at equilibrium.

Molecular machine: molecular assembly that can perform a task through the controlled mechanical actuation of its elementary parts under an appropriate stimulus.

Molecular motor: molecule capable of repetitive directional motion when fueled with a source of energy. There can be linear molecular motors, like molecular walkers, or rotary molecular motors, like motors based on overcrowded alkenes. The unidirectionality is provided by a 'ratcheting' mechanism contained within the molecular motor.

Photothermal effect: phenomenon where a material emits heat after being excited by light irradiation.

Ratchet: macroscopically, a ratchet is a device with asymmetric teeth that bias its continuous motion in one preferential direction (because preventing motion in the opposite direction). By analogy, at the nanoscopic scale in Brownian environment, ratcheted energy profiles are asymmetric and can therefore drive the net unidirectional motion of a system when pumping energy from their environment. In particular, mechanically active molecular systems can make use of such ratcheting strategies to continuously move a particle up to an energy gradient.

\section{Highlights}

A wide variety of stimuli-responsive materials has been recently developed. In particular, the integration of thermodynamic or photodynamic molecular switches in appropriate materials can trigger their macroscopic actuation, possibly in combination with oscillating phenomena.

Most of these systems, however, cannot progressively perform work on their environment because they lack spatial asymmetry in their actuation trajectory. Consequently, any work done in one direction is cancelled once the systems return to their initial state. To create 
actuators that can produce continuous work, ratcheting strategies must be implemented to break the spatial symmetry during their operation.

Macroscopic ratchets can be designed by coupling the whole material with an engineered asymmetric environment during its actuation. Moreover, ratcheting mechanisms can also be implemented in dissipative systems at nanoscale by breaking the spatial symmetry of molecular switches' energy profile, thereby accessing molecular motors.

\section{Outstanding Questions}

Will the integration of molecular motors in macroscopic materials be limited to a few and very specific examples, or can it be implemented by the design of more general and rationalized approaches? In particular, how to preserve - or even amplify -, up to the macroscopic scale, the dissipative out-of-equilibrium ratcheting actuation of a molecular motor when integrated in a material?

Which efficiency can be reached by these systems, in terms of energy conversion, motion's trajectory, workload, speed of actuation, and power? Would these materials present clear advantages compared to simpler switching materials in terms of functioning autonomy and work production?

Are chemically fueled molecular motors capable of integration in actuators? Although famous examples in biology teach us that this is physically possible, how can we simplify enough artificial systems to make them accessible to synthetic chemists while keeping enough functional efficiency?

How to couple such molecularly motorized materials with appropriate devices in order to transduce their mechanical actuation in other types of energy? How to integrate them with other molecular elements (or macroscopic segments) to access multitasking materials? How to engineer them and to interface them with their environment in order to control them and/or to generate autonomous feedback loops leading to adaptation? Can we push such systems sufficiently far away from equilibrium to generate self-organizing emergent behaviors (reaching for instance bifurcation points in their trajectories)? 
Can we make such materials cheap and sustainable enough for useful applications, in particular by simplifying their chemical structures and by improving their resistance to fatigue?

What could be the potential applications of such materials going from energy storage and catalysis, to soft robotics and medicine? Can they become essential part of more complex intelligent materials?

\section{Acknowledgements}

We thank the European Commission's Horizon 2020 Programme as part of the MSCA-ITN project ArtMoMa under grant no. 860434, the FET-Open project MAGNIFY under grant no. 801378, the LabEx CSC, the CNRS, and the University of Strasbourg. We wish to thank Prof. Christian Gauthier for fruitful discussions.

\section{Conflict of interests}

The authors declare no conflict of interests.

\section{References}

1 Schliwa, M. and Woehlke, G. (2003) Molecular motors. Nature 422, 759-765

2 Lindemann, C.B. and Lesich, K.A. (2010) Flagellar and ciliary beating: the proven and the possible. J. Cell Sci. 123, 519-528

3 Endow, S.A. et al. (2010) Kinesins at a glance. J. Cell Sci. 123, 3420-3424

4 Moulin, E. et al. (2020) From Molecular Machines to Stimuli-Responsive Materials. Adv. Mater. 32, 1906036

5 Giuseppone, N. and Walther, A. (2021) Out-of-Equilibrium (Supra)molecular Systems and Materials: An Introduction. In Out-of-Equilibrium (Supra)molecular Systems and Materials pp. 1-19, Wiley

6 Dattler, D. et al. (2020) Design of Collective Motions from Synthetic Molecular Switches, Rotors, and Motors. Chem. Rev. 120, 310-433

7 Beebe, D.J. et al. (2000) Functional hydrogel structures for autonomous flow control inside microfluidic channels. Nature 404, 588-590 
8 Vázquez-González, M. and Willner, I. (2020) Stimuli-Responsive Biomolecule-Based Hydrogels and Their Applications. Angew. Chem. Int. Ed. 59, 15342-15377

9 Shi, J. et al. (2020) Responsive DNA-Based Supramolecular Hydrogels. ACS Appl. Bio Mater. 3, 2827-2837

10 Lu, C.-H. et al. (2015) Multitriggered Shape-Memory Acrylamide-DNA Hydrogels. J. Am. Chem. Soc. 137, 15723-15731

11 Yu, X. et al. (2016) Orthogonal Dual-Triggered Shape-Memory DNA-Based Hydrogels. Chem. Eur. J. 22, 14504-14507

$12 \mathrm{Hu}$, Y. et al. (2016) Reversible Modulation of DNA-Based Hydrogel Shapes by Internal Stress Interactions. J. Am. Chem. Soc. 138, 16112-16119

13 Bruns, C.J. and Stoddart, J.F. (2016) The Nature of the Mechanical Bond, John Wiley \& Sons, Inc.

14 Sluysmans, D. and Stoddart, J.F. (2019) The Burgeoning of Mechanically Interlocked Molecules in Chemistry. Trends Chem. 1, 185-197

15 Goujon, A. et al. (2017) Bistable [ c 2] Daisy Chain Rotaxanes as Reversible Muscle-like Actuators in Mechanically Active Gels. J. Am. Chem. Soc. 139, 14825-14828

16 Goujon, A. et al. (2019) [c2]Daisy Chain Rotaxanes as Molecular Muscles. CCS Chem. 1, 83-96

$17 \mathrm{Bi}$, Y. et al. (2020) Smart Bilayer Polyacrylamide/DNA Hybrid Hydrogel Film Actuators Exhibiting Programmable Responsive and Reversible Macroscopic Shape Deformations. Small 16, 1906998

18 Nakahata, M. et al. (2013) Redox-Generated Mechanical Motion of a Supramolecular Polymeric Actuator Based on Host-Guest Interactions. Angew. Chem. Int. Ed. 52, 57315735

19 Aramoto, H. et al. (2020) Redox-responsive supramolecular polymeric networks having double-threaded inclusion complexes. Chem. Sci. 11, 4322-4331

20 Greene, A.F. et al. (2017) Redox-Responsive Artificial Molecular Muscles: Reversible Radical-Based Self-Assembly for Actuating Hydrogels. Chem. Mater. 29, 9498-9508

21 Zhang, Y. et al. (2015) Fabrication of fluorescent holographic micropatterns based on the rare earth complexes using azobenzene-containing poly(aryl ether)s as macromolecular ligands. J. Polym. Sci. Part A Polym. Chem. 53, 936-943

22 Li, J. et al. (2020) Highly Bidirectional Bendable Actuator Engineered by LCST-UCST 
Bilayer Hydrogel with Enhanced Interface. ACS Appl. Mater. Interfaces 12, 5529055298

23 Lee, T.H. and Jho, J.Y. (2018) Temperature-Responsive Actuators Fabricated with PVA/PNIPAAm Interpenetrating Polymer Network Bilayers. Macromol. Res. 26, 659664

24 Dong, L. and Zhao, Y. (2018) Photothermally driven liquid crystal polymer actuators. Mater. Chem. Front. 2, 1932-1943

25 Yang, Y. et al. (2016) Making and Remaking Dynamic 3D Structures by Shining Light on Flat Liquid Crystalline Vitrimer Films without a Mold. J. Am. Chem. Soc. 138, 2118-2121

26 Yang, H. et al. (2015) Near-infrared-responsive gold nanorod/liquid crystalline elastomer composites prepared by sequential thiol-click chemistry. Chem. Commun. 51, 12126-12129

27 Wang, Y. et al. (2020) Repeatable and Reprogrammable Shape Morphing from Photoresponsive Gold Nanorod/Liquid Crystal Elastomers. Adv. Mater. 32, 2004270

28 Liu, L. et al. (2017) Near-Infrared Chromophore Functionalized Soft Actuator with Ultrafast Photoresponsive Speed and Superior Mechanical Property. J. Am. Chem. Soc. $139,11333-11336$

29 Lahikainen, M. et al. (2018) Reconfigurable photoactuator through synergistic use of photochemical and photothermal effects. Nat. Commun. 9, 4148

30 Lahikainen, M. et al. (2020) Tunable Photomechanics in Diarylethene-Driven Liquid Crystal Network Actuators. ACS Appl. Mater. Interfaces 12, 47939-47947

31 Zhang, H. et al. (2020) Viewpoint: Pavlovian Materials-Functional Biomimetics Inspired by Classical Conditioning. Adv. Mater. 32, 1906619

32 Zeng, H. et al. (2020) Associative Learning by Classical Conditioning in Liquid Crystal Network Actuators. Matter 2, 194-206

33 Wang, C. et al. (2019) DNA-Based Hydrogels Loaded with Au Nanoparticles or Au Nanorods: Thermoresponsive Plasmonic Matrices for Shape-Memory, Self-Healing, Controlled Release, and Mechanical Applications. ACS Nano 13, 3424-3433

34 Yoshida, R. et al. (1996) Self-Oscillating Gel. J. Am. Chem. Soc. 118, 5134-5135

35 Masuda, T. et al. (2018) Chemomechanical Motion of a Self-Oscillating Gel in a Protic Ionic Liquid. Angew. Chem. Int. Ed. 57, 16693-16697

36 Yoshimura, K. et al. (2020) Autonomous oil flow generated by self-oscillating polymer 
gels. Sci. Rep. 10, 12834

37 Kim, Y.S. et al. (2017) Recent developments in self-oscillating polymeric systems as smart materials: from polymers to bulk hydrogels. Mater. Horizons 4, 38-54

38 Yoshida, R. and Uesusuki, Y. (2005) Biomimetic Gel Exhibiting Self-Beating Motion in ATP Solution. Biomacromolecules 6, 2923-2926

39 Gelebart, A.H. et al. (2017) Mastering the Photothermal Effect in Liquid Crystal Networks: A General Approach for Self-Sustained Mechanical Oscillators. Adv. Mater. 29,1606712

40 Vantomme, G. et al. (2018) Self-sustained actuation from heat dissipation in liquid crystal polymer networks. J. Polym. Sci. Part A Polym. Chem. 56, 1331-1336

41 Vantomme, G. et al. (2021) Coupled liquid crystalline oscillators in Huygens' synchrony. Nat. Mater. DOI: 10.1038/s41563-021-00931-6

42 Wei, W. et al. (2018) Phototriggered Selective Actuation and Self-Oscillating in DualPhase Liquid Crystal Photonic Actuators. Adv. Opt. Mater. 6, 1800131

43 Kathan, M. and Hecht, S. (2021) Photoswitchable Components to Drive Molecular Systems Away from Global Thermodynamic Minimum by Light1. In Out-of-Equilibrium (Supra)molecular Systems and Materials pp. 275-304, Wiley

44 Boelke, J. and Hecht, S. (2019) Designing Molecular Photoswitches for Soft Materials Applications. Adv. Opt. Mater. 7, 1900404

45 Hou, J. et al. (2021) Photo-responsive Helical Motion by Light-Driven Molecular Motors in a Liquid-Crystal Network. Angew. Chem. Int. Ed. 60, 8251-8257

46 Bandara, H.M.D. and Burdette, S.C. (2012) Photoisomerization in different classes of azobenzene. Chem. Soc. Rev. 41, 1809-1825

47 Pilz da Cunha, M. et al. (2019) Unravelling the photothermal and photomechanical contributions to actuation of azobenzene-doped liquid crystal polymers in air and water. J. Mater. Chem. C 7, 13502-13509

48 Pilz da Cunha, M. et al. (2020) Bioinspired light-driven soft robots based on liquid crystal polymers. Chem. Soc. Rev. 49, 6568-6578

49 Koshima, H., ed. (2020) Mechanically Responsive Materials for Soft Robotics, Wiley.

50 Pang, X. et al. (2019) Photodeformable Azobenzene-Containing Liquid Crystal Polymers and Soft Actuators. Adv. Mater. 31, 1904224

$51 \mathrm{Wu}, \mathrm{Y}$. et al. (2020) Liquid-Crystalline Soft Actuators with Switchable Thermal 
Reprogrammability. Angew. Chem. Int. Ed. 59, 4778-4784

52 Verpaalen, R.C.P. et al. (2020) Liquid Crystal Networks on Thermoplastics: Reprogrammable Photo-Responsive Actuators. Angew. Chem. Int. Ed. 59, 4532-4536

53 Wani, O.M. et al. (2019) An Artificial Nocturnal Flower via Humidity-Gated Photoactuation in Liquid Crystal Networks. Adv. Mater. 31, 1805985

54 Xiao, Y.-Y. et al. (2021) Desynchronized liquid crystalline network actuators with deformation reversal capability. Nat. Commun. 12, 624

55 Wani, O.M. et al. (2017) A light-driven artificial flytrap. Nat. Commun. 8, 15546

56 Wang, D. et al. (2018) Photoswitchable Azobenzene/Cyclodextrin Host-Guest Complexes: From UV- to Visible/Near-IR-Light-Responsive Systems. ChemPhotoChem 2, $403-415$

57 Iwaso, K. et al. (2016) Fast response dry-type artificial molecular muscles with [c2]daisy chains. Nat. Chem. 8, 625-632

58 Takashima, Y. et al. (2018) A Photoresponsive Polymeric Actuator Topologically CrossLinked by Movable Units Based on a [2]Rotaxane. Macromolecules 51, 4688-4693

59 Klajn, R. (2014) Spiropyran-based dynamic materials. Chem. Soc. Rev. 43, 148-184

60 Satoh, T. et al. (2011) Fast-reversible light-driven hydrogels consisting of spirobenzopyran-functionalized poly(N-isopropylacrylamide). Soft Matter 7, 8030

61 Li, C. et al. (2020) Light-Driven Expansion of Spiropyran Hydrogels. J. Am. Chem. Soc. $142,8447-8453$

62 Li, C. et al. (2020) Supramolecular-covalent hybrid polymers for light-activated mechanical actuation. Nat. Mater. 19, 900-909

63 Kobatake, S. et al. (2007) Rapid and reversible shape changes of molecular crystals on photoirradiation. Nature 446, 778-781

64 Irie, M. et al. (2014) Photochromism of Diarylethene Molecules and Crystals: Memories, Switches, and Actuators. Chem. Rev. 114, 12174-12277

65 Nakagawa, Y. et al. (2019) Photosalient Effect of Diarylethene Crystals of Thiazoyl and Thienyl Derivatives. Chem. - A Eur. J. 25, 7874-7880

66 Fujimoto, A. et al. (2020) Photoinduced swing of a diarylethene thin broad sword shaped crystal: a study on the detailed mechanism. Chem. Sci. 11, 12307-12315

67 Pezzato, C. et al. (2017) Mastering the non-equilibrium assembly and operation of molecular machines. Chem. Soc. Rev. 46, 5491-5507 
68 Aprahamian, I. (2020) The Future of Molecular Machines. ACS Cent. Sci. 6, 347-358

69 Francis, W. et al. (2017) Spiropyran based hydrogels actuators-Walking in the light. Sensors Actuators B Chem. 250, 608-616

70 Li, C. et al. (2020) Supramolecular-covalent hybrid polymers for light-activated mechanical actuation. Nat. Mater. 19, 900-909

71 Yamada, M. et al. (2008) Photomobile Polymer Materials: Towards Light-Driven Plastic Motors. Angew. Chem. Int. Ed. 47, 4986-4988

72 Terao, F. et al. (2012) Light-Driven Molecular-Crystal Actuators: Rapid and Reversible Bending of Rodlike Mixed Crystals of Diarylethene Derivatives. Angew. Chem. Int. Ed. $51,901-904$

73 Pilz da Cunha, M. et al. (2020) A Soft Transporter Robot Fueled by Light. Adv. Sci. 7, 1902842

74 Palagi, S. et al. (2016) Structured light enables biomimetic swimming and versatile locomotion of photoresponsive soft microrobots. Nat. Mater. 15, 647-653

75 Kim, Y.S. et al. (2015) Thermoresponsive actuation enabled by permittivity switching in an electrostatically anisotropic hydrogel. Nat. Mater. 14, 1002-1007

76 Yuan, Y. et al. (2018) Self-assembled nematic colloidal motors powered by light. Nat. Commun. 9, 5040

77 Tong, F. et al. (2020) Light-Powered Autonomous Flagella-Like Motion of Molecular Crystal Microwires. Angew. Chem. Int. Ed. DOI: 10.1002/anie.202012417

78 Wilson, M.R. et al. (2016) An autonomous chemically fuelled small-molecule motor. Nature 534, 235-240

79 Kay, E.R. et al. (2007) Synthetic Molecular Motors and Mechanical Machines. Angew. Chem. Int. Ed. 46, 72-191

80 Feringa, B.L. (2001) In Control of Motion: From Molecular Switches to Molecular Motors. Acc. Chem. Res. 34, 504-513

81 Wilcken, R. et al. (2018) Complete Mechanism of Hemithioindigo Motor Rotation. J. Am. Chem. Soc. $140,5311-5318$

82 Eelkema, R. et al. (2006) Molecular machines: Nanomotor rotates microscale objects. Nature 440, 163

83 Chen, J. et al. (2018) Artificial muscle-like function from hierarchical supramolecular assembly of photoresponsive molecular motors. Nat. Chem. 10, 132-138 
84 Leung, F.K.-C. et al. (2018) Supramolecular Packing and Macroscopic Alignment Controls Actuation Speed in Macroscopic Strings of Molecular Motor Amphiphiles. J. Am. Chem. Soc. 140, 17724-17733

85 Leung, F.K.-C. et al. (2019) Dual-Controlled Macroscopic Motions in a Supramolecular Hierarchical Assembly of Motor Amphiphiles. Angew. Chem. Int. Ed. 58, 10985-10989

86 Li, Q. et al. (2015) Macroscopic contraction of a gel induced by the integrated motion of light-driven molecular motors. Nat. Nanotechnol. 10, 161-165

87 Mariani, G. et al. (2020) Structural properties of contractile gels based on light-driven molecular motors: a small-angle neutron and X-ray study. Soft Matter 16, 4008-4023

88 Foy, J.T. et al. (2017) Dual-light control of nanomachines that integrate motor and modulator subunits. Nat. Nanotechnol. 12, 540-545

89 Orlova, T. et al. (2018) Revolving supramolecular chiral structures powered by light in nanomotor-doped liquid crystals. Nat. Nanotechnol. 13, 304-308 